\title{
Towards Correct and Safe Diagnosis of Specific Learning Disorder in Preschool Age. The perspective of Early Multi-collector Diagnostic Approaches. A Pilot Study
}

\section{Victoria Zakopoulou ${ }^{1 *}$, Katerina D Tzimourta ${ }^{2,5}$, Georgios Ntritsos ${ }^{3,4}$, Alexandros T Tzallas ${ }^{4}$, Markos G Tsipouras ${ }^{5}$, Loukas G Astrakas ${ }^{2}$, Pavlos Christodoulides ${ }^{1}$, Ioannis Paliokas ${ }^{6}$, Vassilis Zakopoulos ${ }^{7}$ and Nikolaos Giannakeas ${ }^{4}$}

${ }^{1}$ Department of Speech and Language Therapy, Laboratory of New Approaches in Communication Disorders, University of Ioannina, Ioannina, Epirus, Greece

${ }^{2}$ Medical Physics Laboratory, School of Medicine, University of Ioannina, Ioannina, Greece

${ }^{3}$ Department of Hygiene and Epidemiology, School of Medicine, University of Ioannina, Ioannina, Greece

${ }^{4}$ Department of Informatics and Telecommunications, School of Informatics and Telecommunications, University of Ioannina, Ioannina, Greece ${ }^{5} 50100$, Kozani, Greece

${ }^{6}$ Information Technologies Institute, Centre of Research and Technology - Hellas $6^{\text {th }}$ km Harilaou - Thermi, Thessaloniki, Greece

${ }^{7}$ Department of Accounting and Finance, School of Administrative, Economics, and Social Sciences, University of West Attica, Athens, Greece

*Corresponding Author: Victoria Zakopoulou, Department of Speech and Language Therapy, Laboratory of New Approaches in Communication Disorders, University of Ioannina, Ioannina, Epirus, Greece.
Received: April 14, 2021

Published: May 17, 2021

(C) All rights are reserved by Victoria

Zakopoulou., et al.

\section{Abstract}

Background: The wide range of terminology, multiple diagnostic criteria, and multifarious basis of Specific Learning Disorder (SLD) constitutes a new reality in the complex entity of SLD. In this manuscript, we address this issue and present the findings of a pilot study concerning the early diagnosis of SLD, while strongly emphasizing the necessity of integrating and testing a vast range of data from multiple domains and skills to achieve correct and safe early diagnosis of SLD.

Materials and Methods: For this purpose, statistical techniques were implemented in a well-structured methodological approach, as follows: (a) a cluster of adequate diagnostic procedures to determine the early extent of specified difficulties, (b) targeted data clustering techniques to identify clusters in the data, and (c) the Use Case method for the configuration of individualized diagnostic profiles.

Results: Through a data analysis schema, several variables were reported as significant, clustering the participants according to their strengths and weaknesses, while strong interactions between specific factors were highlighted in the background of SLD.

Conclusion: The findings of the study enhance the core argument of this pilot study that an "ever-expanding model" should be considered as the most reliable source for a comprehensive early diagnosis of SLD.

Keywords: Specific Learning Disorder; Early Diagnosis; Multifactorial Approach; Early Intervention; Clustering 


\section{Abbreviations}

SLD: Specific Learning Disorder; EDIT: Early Dyslexia Identification Test; WPPSI-IIIGR: Wechsler Preschool and Primary Scale of Intelligence-Greek Edition; CA: Cluster Analysis; CBCL 11/2-5: Child Behavior Checklist for Ages $1 \frac{1}{2}$ to 5

\section{Introduction}

Specific Learning disorder (SLD) refers to a multi-system deficit based on complex system of interactions between more than one genetic, neurological, cognitive, social, and emotional dysfunctions [1].

Each time, new dimensions of the deficit are uncovered and reveal the complexity of the problem and its wide range symptoms and extensions, thus increasing the necessity of implementing comprehensive approaches to SLD, based on interdisciplinary and multifactorial studies (neurological, biological, psychosocial, learning, and neuro-cognitive) [2].

According to the classification system of mental health disorders for children and adults (DSM-5) [3], SLD refers to specific deficits in the individual's ability to perceive or process information efficiently and accurately. The heterogeneity in terms of challenge, degree of severity, aggravating factors, and degree of response to intervention and the multifactorial accompanying characteristics of SLD, render both diagnosis and intervention extraordinarily complex.

To this, children under the age of five with neurodevelopmental disorders may experience problems with speech, motor skills, learning or other neurological functions [4]. People often focus only on one aspect of these problems, considering that it should be "just autism" or "only Attention Deficit Hyperactivity Disorder (ADHD and so on, underestimating the actual situation. However, over the years evidence has shown that many disorders may coexist [4]. For example, many disorders such as: ADHD, Oppositional Defiant Disorder (ODD), Specific Learning Disorder (SLD), Language Disorder (LD), and Intellectual Disability (ID) may coexist in children with different combinations. Most of the abovementioned disorders have been studied mainly at school age and some of them have been studied in preschool age. However, even when develop- mental disorders occur during pre-school age, many cases do not get the attention of specialists before school age. As a result, 10\% of children facing similar problems do not reflect the number of children who are accurately diagnosed and treated at pre-school age [5].

In addition, it is extremely important to comprehend that these are developmental disorders, meaning that the symptomatology fluctuates with time and response to intervention [6]. In this way, the diagnosis depends on the theoretical approach of the specialist, which often happens to be different from one individual to another. As a result, different types of disorders are created, or it becomes difficult to accept the comorbidity of disorders. This fact raises concerns about the accuracy of diagnoses and thus, of the appropriate interventions [7].

Based on several studies [8] the biological origin of SLD constitutes of a complex clinical entity characterized by dysfunctional interactions between genetic, epigenetic, neurophysiological, and environmental factors that evoke cognitive abnormalities and behavioral problems disrupting the process of learning academic skills (reading, writing, spelling, mathematics). As a result, it becomes obvious that these interactions are maintained by endogenous chain-like effects between them (Figure 1).

Aiming to identify the role and the significance of each factor in this chainlike, long-term, and often conflicting translational approaches emphasize different causes such as genetic, neurological, cognitive, behavioral and psychoemotional [9].

The constitutional domains of the SLD's endo-phenotype and relative dysfunctions

The chain-like influences address separate domains, reflecting the different components of the SLD endo-phenotype.

\section{Biological domain}

The study of quantitative genetic research indicates that the genes are responsible for the high heritability of learning disabilities and abilities [10]. The large genetic correlations within and between learning disabilities and abilities suggest that genes with general effects are considered important for molecular genetics research. 


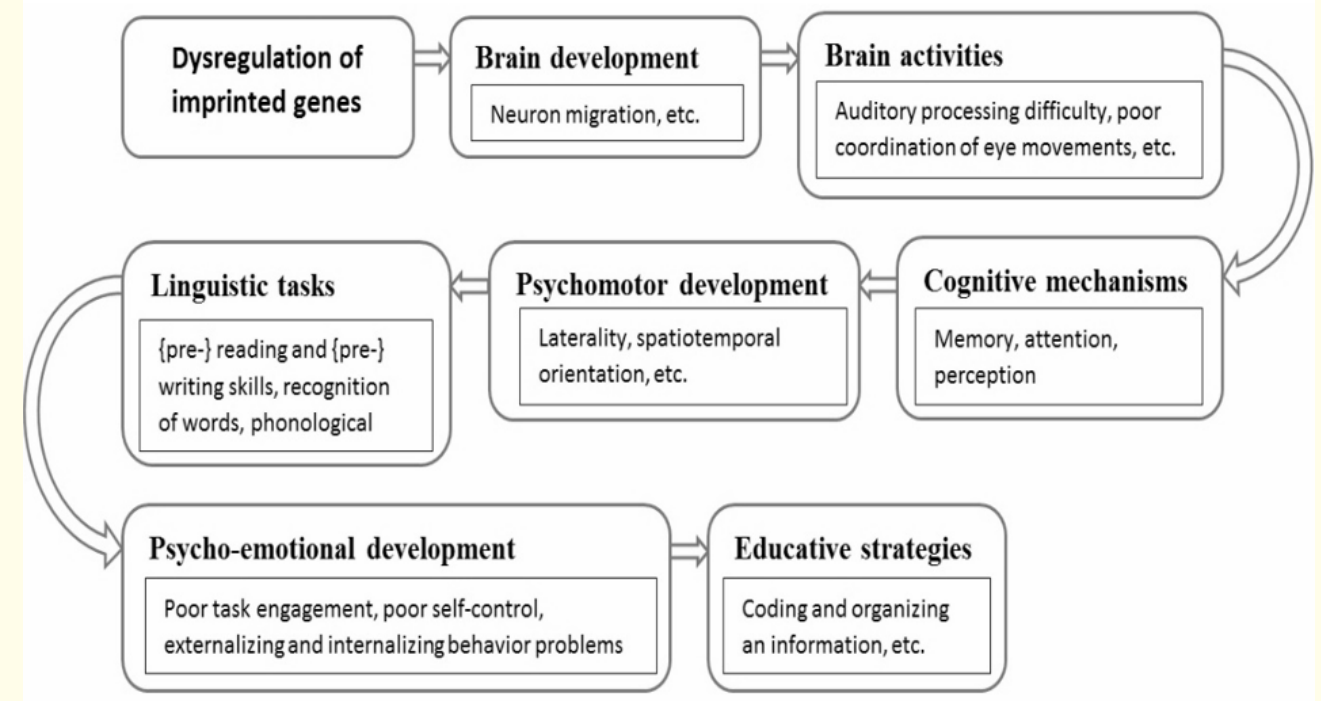

Figure 1: Chainlike influences between particular factors that constitute the sub-domains of SLD.

Twin and family-based studies show that genes are responsible for the high heritability of SLD that both genetic and environmental factors underlie the construction of SLD in strong complexity [11]. The study of genes and their complex interaction with learning difficulties constitutes a new research area [12] and has provided significant results in early diagnosis. This could facilitate preschool intervention prior to the manifestation of reading deficits and might aid diagnosis at later ages. Multiple linkage analyses identified at least five genes (DYX1C1, ROB01, DCDC2, TTRAP, and KIAA0319), several varieties of which those could be accused as susceptible factors of the onset of SLD and its continuity in later life [13]. Similarly, twelve other genes (FOXP2, CNTNAP2, DOCK4, GTF2I, GRIN2B, SLC2A3, ATP2C2, CMIP, PCNT, DIP2A, S100B and PRMT2) have been correlated with reading [10].

Techniques of molecular genetics, such as Cytogenetic analysis, FISH analysis, Microsatellite DNA analysis, Molecular analysis, and linkage analysis in families, provide the potential of diagnosing small abnormalities in chromosomes that cannot be diagnosed just with classic ideogram.

\section{Neurological domain}

There is evidence that SLD is characterized by specific neurological deficits and possibly by neuroanatomical variations [14]. Some of these neuroanatomical abnormalities include thinner cortex in primary auditory cortex (Herschel's gyrus) and visual areas in preschoolers [8]. In school-age children with double-deficit, reduced activation has been recorded in the left temporo-parietal region, including cerebellar lobules, Inferior Parietal Lobule (IPL), and left Inferior Frontal Gyrus (IFG) [15]. Similarly, hypoactivation has been observed within left temporo-parietal and occipito-temporal areas [16], and distinct asymmetry patterns in frontal and parietal regions [17] considered as responsible for storage and manipulation of phonological information. In line with the above, it is estimated that about $50 \%$ of children with epilepsy shows schooling difficulties, while benign childhood epilepsy with centrotemporal spikes (BECTS) is the most common partial epilepsy syndrome in pediatric patients $[14,18]$.

Today, many studies attempt to study and record children's brain activity through electrophysiological [19] and brain imaging techniques $[10,15,16]$. Recent studies $[8,16]$ showed that disorders regarding the activity and structure of the left temporaloccipital brain area (including thinner cortex in Heschl's gyrus, lingual gyrus, medial frontal gyrus, middle cingulate gyrus), and cerebellar (maturational delay or asymmetry) play a major role in the recognition of words as well the accomplishment of phonological tasks. Another promising line of using electrophysiological 
Towards Correct and Safe Diagnosis of Specific Learning Disorder in Preschool Age. The perspective of Early Multi-collector Diagnostic Approaches. A Pilot Study

measures (EEG, ERPs) [9] is the online tracking of processing impairments of speech and language shown by a large percentage of SLD children. In addition, fine binocular coordination of saccades is considered an overly sensitive marker of global brain function, cortical maturation, particularly involving the magnocellular stream and the cerebellum [22].

All these observations provide a new basis to understand whether the various SLD-related behavioral and neural differences derive from low-level adaptation-related deficits, and also suggest that this disorder may be characterized by a general impairment in mechanisms for short-term plasticity and rapid perceptual learning. To achieve a more comprehensive understanding of suspicious factors of reading difficulties, an emerging body of approaches on SLD $[23,24]$ tend to examine both imaging and genetic data in a common neurobiological mapping of SLD. These advances have showed great potential and played a key role in understanding the neuroanatomical and cognitive basis of SLD; thus addressing, the neural signature of SLD.

\section{Cognitive domain}

Several studies suggest that the reading deficit is related to problems of phonological processing [8,25]. Frequently, SLD is characterized by deficits in phonological memory and slow progress in the development and production of: (a) phonological codes of sounds (deficiency in auditory perception and discrimination of phonemes), (b) phoneme-grapheme correspondence [26], and (c) semantic recognition of words and non-words [27]. Moreover, one of the causes connected with SLD is the primary sensory process concerning visual and auditory process [28,29]. However, more surveys are demanded to investigate and discuss the prevalence of causative role of sensory deficits in the onset of SLD. In a recent study, Goswami (2011) [30] proposed that in the case of SLD, specific mechanisms in the auditory cortex dealing with temporal information are impaired that normally contribute to the perception of stress and rhythm, leading to phonological deficits. Furthermore, a vast number of approaches and measurements are used to test early difficulties in cognitive and linguistic mechanisms, such as: (a) phonological awareness, (b) phonological memory, (c) verbal working memory, (d) visual short term memory, (e) rapid naming language development, (f) pragmatic skills, (g) communication, problem solving, (h) reasoning and numeracy and (i) knowledge and understanding of the world (e.g. the Early Years Foundation Profile Stage (EYFS) [31].

\section{Socio-psycho-emotional domain}

It is evident that developmental language, communication, and literacy skills affect areas of socio-emotional functioning. Anxiety, depression, stressful events, emotional trauma, and other conditions affecting concentration make learning process more challenging [32]. Moreover, a variety of disorders have been found to affect the way verbal and non-verbal information is understood, organized, stored, acquired, and expressed. If SLD is left untreated, the frustration and difficulties caused by learning difficulties both at home and school, will create stress and depression, leading to low self-esteem [33]. However, the interaction between psychological and learning difficulties is extremely complicated, and further research is needed to clarify this kind of interaction.

Aiming to understand the multifaceted complexity of SLD, an emerging body of studies supports that a dimensional view of all the relative functions and interactions between these various components is required [34].

\section{The early phenotypic characteristics}

The necessity of highlighting the interactions mentioned above, as early as possible, is also considered as both indicative and demanding. In light of the above, it becomes imperative to organize and implement comprehensive diagnostic methods and appropriate intervention at the preschool age, before these difficulties turn into specific symptoms of SLD in primary school, thereby losing valuable time. Studies have reported that clinical indications of future learning disorders can already be detected from two-to-six years of age [16].

Several studies [35] focus on the psycho-cognitive correlates of dyslexia and the neural correlates of speech sound processing and word recognition. A longitudinal project on the precursors of dyslexia [36], aimed on early intervention focusing on reading fluency using a cross-linguistic approach, co-morbidity with dyscalculia or other learning difficulties and the development of dynamic tests (especially concerning the acquisition of letter knowledge). In a 
Towards Correct and Safe Diagnosis of Specific Learning Disorder in Preschool Age. The perspective of Early Multi-collector Diagnostic Approaches. A Pilot Study

recent publication [10] an interdisciplinary neuroimaging-genetics approach was proposed, aiming to elucidate the underlying mechanisms of SLD and consequently, to lead to the early intervention.

It has been reported that if children with SLD attend remediation programs starting in preschool and first grade, they will show better development because the intervention time needed is shorter $[8,16]$. In that direction, low-resource programs, such as playing action video games without phonological or orthographic training [37] or training through a whole-body sensorimotor system, could improve their reading and attention ability. At this age, the brain's high plasticity may enable SLD children to reach the skills of typically developing children [38].

The perspective of early multi-collector diagnostic models: The big data concept

To date, studies and clinical work have shown great interest in analyzing factors from specific domains, which are related to SLD. However, the correlation between all the above-mentioned factors remains vague, whereas it is significantly valuable for an integrated interpretative approach and therefore for the diagnosis and early intervention of SLD.

Traditional data processing techniques seem to be inadequate due to the size and complexity of these data. To seize the large potential of data fusion and big data analysis, the last appears to be a powerful solution that will shed light on an explicit early diagnosis of SLD. On the other hand, contemporary technological advances towards Big Data Analytics and data mining techniques can help Health organizations, research institutes and independent researchers on processes related to collecting and analyzing large sets of data with the purpose to discover hidden patterns and other useful information. Analysts will be able to model heterogeneous data collections and to identify the data that is most important to the SLD diagnosis and the related decision making before intervention planning. Moreover, the new knowledge which comes from analyzing the integrated data collections will be stored in a large knowledge base and be reused. In addition, we need reduced data models to face the increasing complexity of the existing and new data collections, which requires increased performance and processing power.
For the challenges defined in SLD, a systematic comparison between various evaluation results to validate new early diagnostic techniques is highly needed. Currently, data and new knowledge remain scattered in peripheral and disconnected repositories making management and access difficult if not impossible. Specifically, a large database of systematic recording, classification and co-evaluation of all data and their possible associations and interactions should be created to control all forms of manifestation and interaction. Thus, it will interpret all the included the data and lead to safe diagnoses and effective interventions in SLD.

\section{Objectives}

In this manuscript, we propose the use of an "ever-expanding model" for a comprehensive and multifaceted early diagnosis of SLD. Accordingly, we emphasize the expectation of developing an early multi-collector diagnostic model that will be based on data derived from multiple domains. It is a concept paper, in which we present the findings of a pilot study of preschoolers diagnosed 'at risk' for SLD.

Considering the vast array of different and often controversial approaches of the SLD's multidimensional diagnosis, the aim of this study was to: (a) discuss the strong complexity between weaknesses in multiple domains that are correlated with SLD and (b) emphasize the lack of effective assessments for a comprehensive early diagnosis of SLD, such as the Big Data analysis could be produced or harnessed (it seems to be the imminent challenge in human sciences). For this purpose, we attempted to construct a model to collect and classify a vast number of methods and data. Utilizing a data analysis schema, we assessed these methods and analyze all the accessible data to create correct early diagnostic profiles.

The proposed research protocol was followed, aiming to examine, manipulate, and translate the role of specific factors in a chain of interactions in the early occurrence of SLD, as following: (a) visual-spatial abilities, (b) cognitive mechanisms/working memory, (c) language acquisition, (d) phonological awareness, (e) psychoemotional development, and (f) intellectual abilities.

To investigate whether this model contribute to clarify all possible multifarious interactions between competent mechanisms of 
the structure of SLD, we attempted to answer the following questions:

- Do specific dysfunctions in one or more cognitive, socio-psycho-emotional, and linguistic domains indicate the early onset of SLD?

- Could an early multi-collector diagnostic model be suggested as adequate to underline, co-evaluate, and translate the complex interactions between the above domains in order safe diagnostic profiles to be created?

- Which of the processed data t could be considered enough for a precise configuration of all the underlined endo-phenotypic alterations in the above domains, even at early developmental stages?

It is important to stress here, that our main concern is, that the procedure suggested in this article aims to assist in offering solutions to this problem's complexity rather than to imply that the use of data for a large number of observations would solve the issues that arise from the use of a multitude of different tests.

\section{Materials and Methods}

In this perspective, we designed a well-structured methodological approach to reach the following three key-milestones: (a) implementation of a cluster of adequate diagnostic checks to determine the early extent of difficulties, (b) identification of clusters in the data using data clustering techniques, and d) configuration of individualized diagnostic profiles using of the Use Case method.

\section{Participants}

All the participants were children attending kindergarten and their parents were asked to sign a consent form allowing their children to participate in the diagnostic procedure. The whole evaluation process was implemented by experts who were blind to the children's allocation. The main inclusion criteria for children's participation in the study were their nationality (Greek), the fact that they were monolingual, their age 5-6 years old (M= 65 months and 20 days) and they were attending kindergarten (the sample was in the 2nd year of kindergarten). Consequently, children with Intellectual Disability, Autistic Spectrum Disorder (ASD), Specific Language Impairment (SLI), and any neurological disorder were excluded from the study, resulting in 20 participants fulfilling the above criteria. The study was conducted during the period between $10 / 05 / 2016$ to $10 / 06 / 2017$ in accordance with the Declaration of Helsinki, and the protocol was approved by the Scientific Committee of the University Hospital of Ioannina (Id. Code: 1-15/4/2016). The whole survey complied with ethics, as adopted by the General Assembly of the World Medical Association [39].

\section{Diagnostic measures}

Four diagnostic measures were implemented namely.

Early Dyslexia Identification Test [40] was used as the main screening test for the definition of children 'at risk' for SLD. By implementing the EDIT test (a standardized screening test of early signs and trends of dyslexia in preschoolers, with mean degree of reliability 0.98 ) we examined three sectors through 8 tasks, targeting to create early developmental dyslexia's profiles, as follows:

- Visual-spatial Abilities (Sketching, Copying shapes, Laterality/Left-right discrimination),

- Grapho-phonological Awareness (Phonemes Composition; Phonemes Discrimination; Name Writing) and

- Working Memory (Phonemes Discrimination, Name Writing, Copying shapes, Visual-verbal correspondence).

ATHINA test [41]: by implementing the ATHINA test (a standardized diagnostic test of difficulties in learning in children aged 5-8 years, with mean degree of reliability 0.85 ) we evaluated four sectors through 14 tasks, targeting to detect difficulties in cognitive, perceptual, psycholinguistic, and motor processes, as follows:

- Verbal Intelligence (verbal correspondence, vocabulary, copying shapes),

- $\quad$ Short-term Sequence Memory (Numbers Memory, Pictures Memory, Shapes Memory),

- Integration of Incomplete Performances (Sentences completion, words completion),

- Grapho-phonological Awareness (Phonemes Discrimination, Phonemes Composition, Grapheme Discrimination), and

- $\quad$ Neuro-psychological Maturity (laterality, left-right discrimination, visual-motor coordination). 
Greek edition of Child Behavior Checklist for Ages $1 \frac{1}{2}$ to 5 (CBCL 11/2-5) [42]: by implementing the CBCL (11/2-5) form we tested three categories of problems, such as: Total Problems, Internalizing, Externalizing, and 7 syndromes: Emotionally Reactive, Anxious, Depressed, Aggressive Behavior, Attention Problems, Somatic Complaints, and Withdrawn, creating profiles classified in normal, borderline and clinical ranges.

Greek edition of Wechsler Preschool and Primary Scale of Intelligence [43]: by implementing WPPSI-IIIGR (only the core subtests for children ages 4.0-7.3 years were considered) we tested four scales through 8 subtest, representing the Verbal IQ (VIQ)
(Information, Vocabulary, and Word Reasoning), the Performance IQ (PIQ) (Block Design, Matrix Reasoning, and Picture Concepts), the Processing Speed Quotient (PSQ) (Coding), and the Full Scale IQ (FSIQ) (Information, Vocabulary, Word Reasoning, Block Design, Matrix Reasoning, Picture Concepts, and Coding).

\section{Diagnostic procedure}

The concept of proposing for implementation an early multicollector diagnostic model towards a comprehensive diagnosis of SLD's multifaceted entity has been formulated, as follows:

- Initially, the data were used to generate several profiles covering all-possible important theoretical aspects (Figure 2).

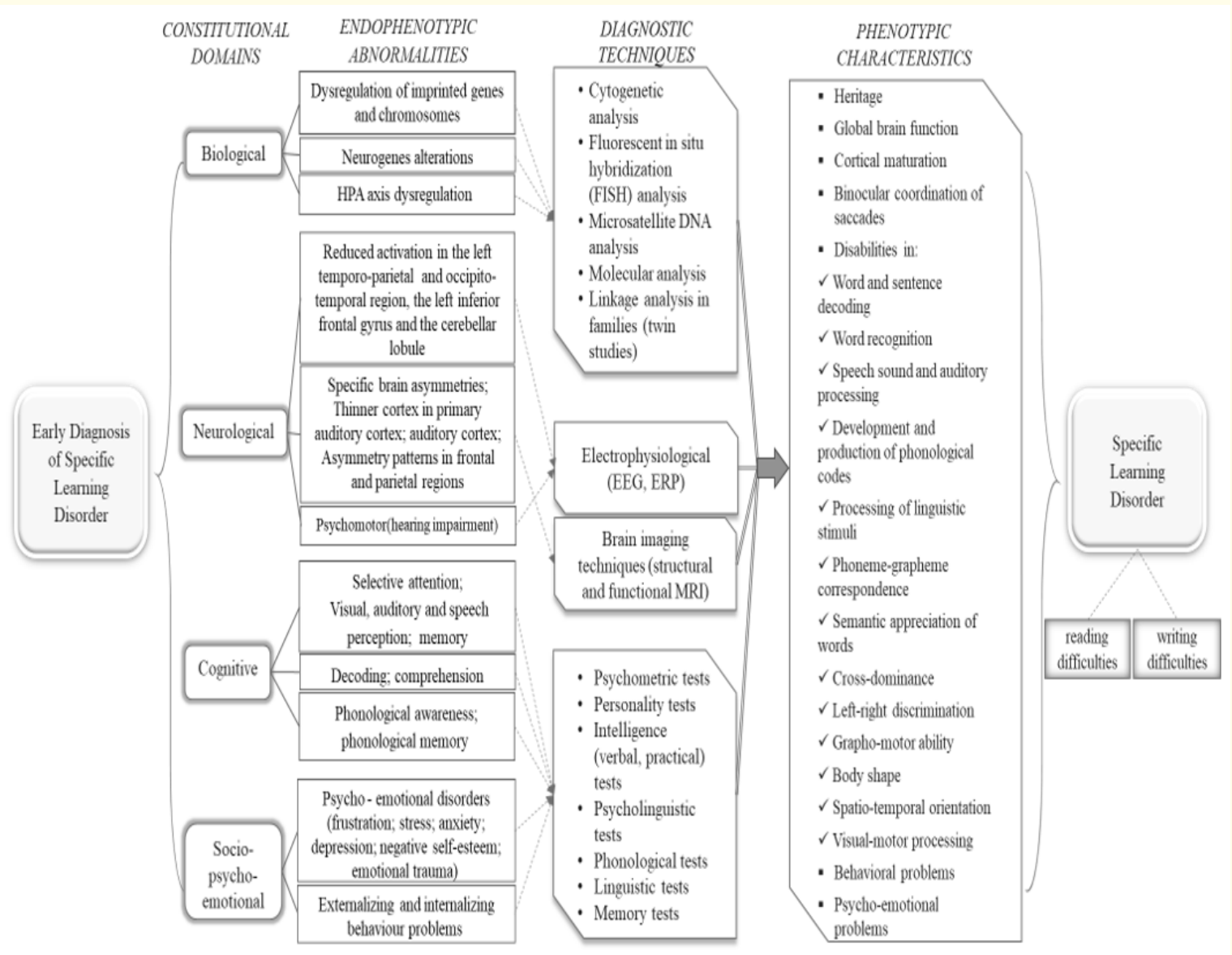

Figure 2: Depiction of the proposed multicollector model for the explicit early diagnosis of SLD. 
It is worth mentioning that in this pilot study we used no data pertained to biological nor to neurological domains, but only to cognitive, language, and socio-psycho-emotional domains. Despite this limitation, the findings of the current survey are strongly recommended for the creation of an early multi-collector diagnostic model.

Secondly, data clustering techniques were used to identify clusters in the data. A dendrogram using average linkage between groups illustrated hierarchical clustering analyses between 20 children examined in specific variables. Each cluster represented an assembly of data with high similarity, while it can be appointed to a SLD, or to the normal case. Thus, each cluster can be recognized as a profile associated to a specific outcome, while the centroid of the cluster is the average value of the profile for each characteristic (i.e. feature extracted from a specific measurement). Since the knowledge extraction is the main goal, association rule mining is one of the most promising options. The main idea was to analyze the data to extract frequent item sets with specific confidence and support. In general, these item sets cover all possible feature combinations. As a result, a database with rules predicting the outcome was created. Classification algorithms were also employed in the data analysis. After the generation (training) of the classification model, it can classify any new, unseen data to a specific class (such as reading difficulties, writing difficulties, either difficulties or no difficulties).

Finally, Unified Modeling Language (UML) Use Case Diagram was used to clarify the proposed database design implementation and capture what the system is supposed to do. It is worth noting, that in this case are also included all the domains that theoretically could be interacted in the occurrence of SLD. The purpose of the diagram was to present the way a candidate for SLD interacts with a sequence of actions.

\section{Statistical analysis}

The statistical analysis was based on the following performance metrics:

- Mahalanobis distance [44] was used to detect outlier cases based on their values in the variables of the EDIT test.
- $\quad$ Cluster Analysis (CA), was used to classify our participants into homogenous groups, using the available information of the variables of interest. Agglomerative hierarchical clustering assumes that each participant starts in its own cluster, and pairs of clusters are merged as one move up the hierarchy. We aimed to explore how different combinations of variables modify the clusters among our participants.

- K-means clustering algorithm was used to assess the accordance of the main case-control dataset with the clusters that created by the combinations of variables. K-means is a partitioning algorithm that implies an a priori known number of clusters (requires the a priori specification of the number of clusters) and distributes the participants into specific clusters depending on which cluster is "nearest" to the participant. All Statistical Analyses were Performed Using IBM SPSS Statistics 26.3.

\section{Results}

The calculation of used Mahalanobis distance for our participants, based on their values on EDIT variables end up with one participant as outlier. As a result, 19 participants were included in CA. Hierarchical CA, using the agglomerative algorithm showed that the addition of variables as they are included in the tests inserted in the model, fragmented our participants in more groups. More specifically, the addition in this order, of EDIT-testing, ATHINA, CBCL1 $1 / 2-5$ and WPPSI III did fragment our participants into progressively more clusters (Figure 3). This fragmentation seems to have arisen since the added tests examined variables different from those of EDIT test, thus leading to new clusters characterized by new dataset.

Using the K-means clustering algorithm we assessed the accordance of the main case-control dataset with the clusters that created by the combinations of variables (Table 1). Based on the EDIT-testing variables, 12 participants (8 with SLD and 4 without) were distributed in the first cluster and 7 (3 with SLD and 4 without) in the second. Adding the ATHINA-testing variables to the EDIT-testing variables, we observed a more vigorous fragmentation of our participants, while 16 participants were distributed in the first cluster (10 with SLD and 6 without) and 3 in the second 


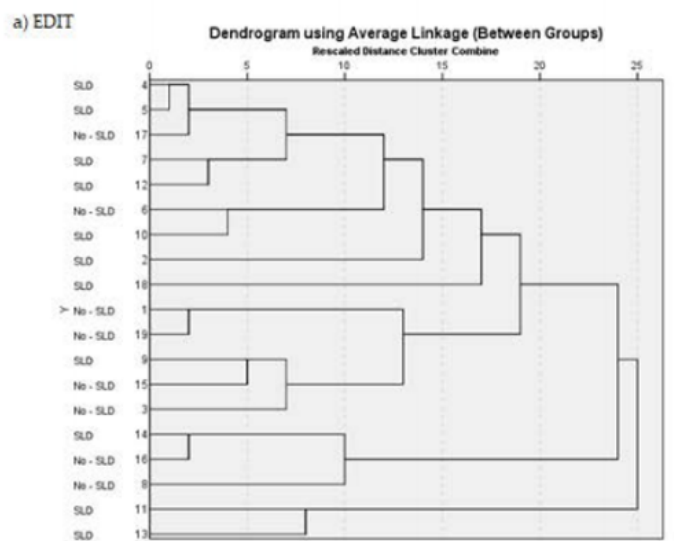

c) $\mathrm{EDT}+\mathrm{ATHINA}+\mathrm{CBCL} 11 \mathrm{z}-5$

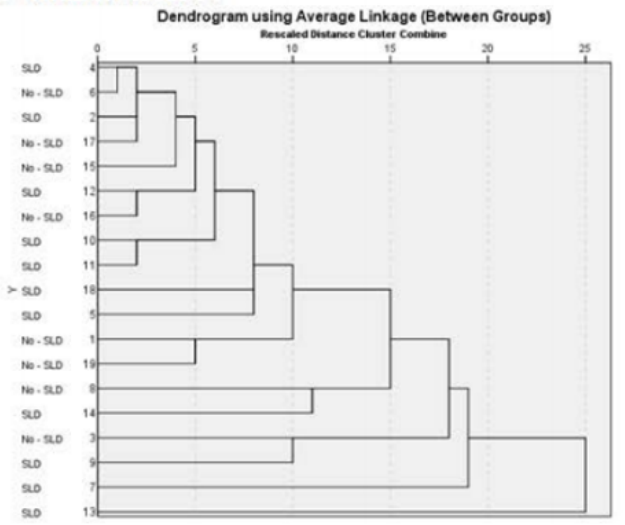

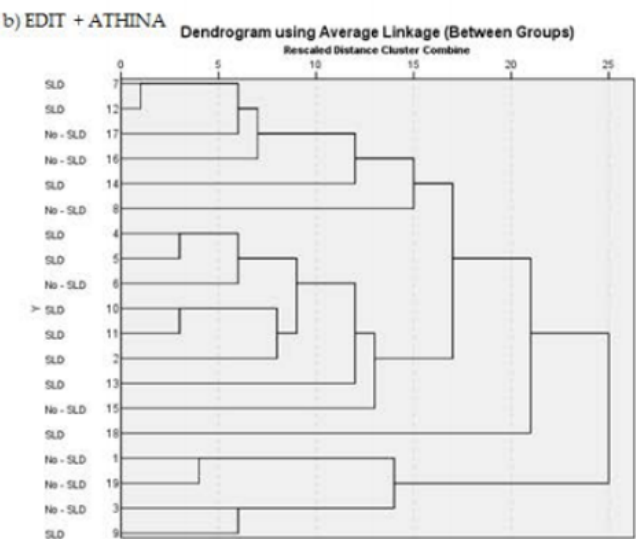

d) EDIT + ATHINA +CBCL11/2-5+ WPSSI- III

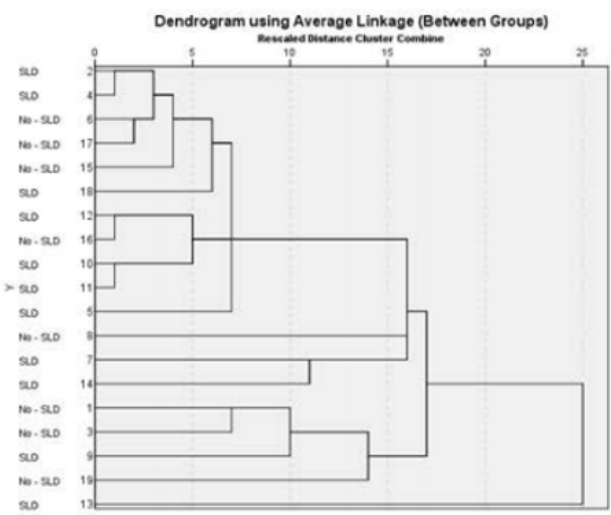

Figure 3: (a, b, c, d): Dendogram Using Average Linkage (between groups).

\begin{tabular}{|c|c|c|}
\hline EDIT & SLD & No-SLD \\
\hline Cluster 1 & 8 & 4 \\
\hline Cluster 2 & 3 & 4 \\
\hline EDIT+ATHINA & & \\
\hline Cluster 1 & 10 & 6 \\
\hline Cluster 2 & 1 & 2 \\
\hline EDIT+ATHINA+CBCL11/2-5 & & \\
\hline Cluster 1 & 7 & 2 \\
\hline Cluster 2 & 4 & 6 \\
\hline EDIT+ATHINA+CBCL1 $1 / \mathbf{2}-\mathbf{5 + W P P S I - I I I ~}$ & & \\
\hline Cluster 1 & 8 & 2 \\
\hline Cluster 2 & 3 & 6 \\
\hline
\end{tabular}

Table 1: Accordance between case-control dataset and K-means CA.
(1 with SLD and 2 without). Moreover, adding CBCL11/2-5 variables in the hierarchical CA we observed a more extreme fragmentation, according to which, 9 participants were distributed in the first cluster (7 with SLD and 2 without) and 10 in the second cluster (4 with SLD and 6 without). The addition of WPPSI-III improved the fragmentation compared to the previous steps. The combination of EDIT, ATHINA, CBCL1 $1 / 2-5$ and WPSSI testing variables distributed 10 participants in the first cluster ( 8 with SLD and 2 without) and 9 participants in the second cluster (3 with SLD and 6 without).

Investigating in depth the above CA results, we evaluated the analysis of variance (ANOVA) table that arises from the K-means clustering algorithm. This one-way ANOVA uses the specific variables as dependent variables and the clusters determined by $\mathrm{K}$ means as a factor. Table 2 presents which specific variables were 
Towards Correct and Safe Diagnosis of Specific Learning Disorder in Preschool Age. The perspective of Early Multi-collector Diagnostic Approaches. A Pilot Study

\begin{tabular}{|c|c|c|c|c|c|c|}
\hline \multirow{2}{*}{$\begin{array}{c}\text { Variables } \\
\text { EDIT }\end{array}$} & \multicolumn{2}{|l|}{ Cluster } & \multicolumn{2}{|l|}{ Error } & \multirow{2}{*}{$\mathbf{F}$} & \multirow{2}{*}{$\mathbf{P}$} \\
\hline & Mean Square & Df & Mean Square & Df & & \\
\hline Sketching & 5.786 & 1 & .718 & 17 & 8.053 & .011 \\
\hline Copy Shapes & .012 & 1 & 1.058 & 17 & .012 & .915 \\
\hline Laterality & .762 & 1 & 1.014 & 17 & .752 & .398 \\
\hline Left-right Discrimination & 3.000 & 1 & .882 & 17 & 3.401 & .083 \\
\hline Name Writing & 8.229 & 1 & .575 & 17 & 14.316 & .001 \\
\hline Phonemes Discrimination & 5.398 & 1 & .741 & 17 & 7.282 & .015 \\
\hline \multicolumn{7}{|l|}{ EDIT+ATHINA } \\
\hline Sketching & 4.961 & 1 & .767 & 17 & 6.469 & .021 \\
\hline Copy Shapes & .073 & 1 & 1.055 & 17 & .069 & .796 \\
\hline Laterality & 4.253 & 1 & .809 & 17 & 5.259 & .035 \\
\hline Left-right Discrimination & .105 & 1 & 1.053 & 17 & .099 & .756 \\
\hline Name Writing & 4.225 & 1 & .810 & 17 & 5.214 & .036 \\
\hline Phonemes Discrimination & 5.786 & 1 & .718 & 17 & 8.053 & .011 \\
\hline Verbal Correspondences & 8.872 & 1 & .537 & 17 & 16.523 & .001 \\
\hline Vocabulary & 3.241 & 1 & .868 & 17 & 3.733 & .070 \\
\hline Pictures Memory & .819 & 1 & 1.011 & 17 & .810 & .381 \\
\hline Shapes Memory & 9.865 & 1 & .479 & 17 & 20.617 & .000 \\
\hline Sentences Completion & 5.867 & 1 & .714 & 17 & 8.221 & .011 \\
\hline Graphemes Discrimination & 1.963 & 1 & .943 & 17 & 2.080 & .167 \\
\hline \multicolumn{7}{|l|}{ EDIT+ATHINA + CBCL1 1 $1 / 2-5$} \\
\hline Sketching & 6.144 & 1 & .697 & 17 & 8.810 & .009 \\
\hline Copy Shapes & .869 & 1 & 1.008 & 17 & .863 & .366 \\
\hline Laterality & 3.873 & 1 & .831 & 17 & 4.660 & .045 \\
\hline Left-right Discrimination & 3.647 & 1 & .844 & 17 & 4.319 & .053 \\
\hline Name Writing & 4.320 & 1 & .805 & 17 & 5.368 & .033 \\
\hline Phonemes Discrimination & 1.488 & 1 & .971 & 17 & 1.532 & .233 \\
\hline Verbal Correspondences & .908 & 1 & 1.005 & 17 & .903 & .355 \\
\hline Vocabulary & .585 & 1 & 1.024 & 17 & .571 & .460 \\
\hline Pictures Memory & .589 & 1 & 1.024 & 17 & .575 & .459 \\
\hline Shapes Memory & 2.338 & 1 & .921 & 17 & 2.538 & .130 \\
\hline Sentences Completion & 2.268 & 1 & .925 & 17 & 2.451 & .136 \\
\hline Graphemes Discrimination & .670 & 1 & 1.019 & 17 & .657 & .429 \\
\hline Emotionally Reactive & 8.817 & 1 & .540 & 17 & 16.321 & .001 \\
\hline Anxious, Depressed & .067 & 1 & 1.055 & 17 & .064 & .804 \\
\hline Somatic Complaints & .236 & 1 & 1.045 & 17 & .226 & .641 \\
\hline Withdrawn & 1.780 & 1 & .954 & 17 & 1.865 & .190 \\
\hline Attention Problems & .900 & 1 & 1.006 & 17 & .895 & .357 \\
\hline Aggressive Behavior & 6.207 & 1 & .694 & 17 & 8.947 & .008 \\
\hline
\end{tabular}


Towards Correct and Safe Diagnosis of Specific Learning Disorder in Preschool Age. The perspective of Early Multi-collector Diagnostic Approaches. A Pilot Study

\begin{tabular}{|c|c|c|c|c|c|c|}
\hline Internalizing Problems & 3.151 & 1 & .873 & 17 & 3.607 & .075 \\
\hline Externalizing Problems & 5.532 & 1 & .733 & 17 & 7.543 & .014 \\
\hline Total Problems & 1.470 & 1 & .972 & 17 & 1.512 & .236 \\
\hline \multicolumn{7}{|c|}{ EDIT+ATHINA+CBCL11/2-5+WPPSI-III } \\
\hline Sketching & .001 & 1 & 1.059 & 17 & .001 & .980 \\
\hline Copy Shapes & .869 & 1 & 1.008 & 17 & .863 & .366 \\
\hline Laterality & 4.188 & 1 & .812 & 17 & 5.155 & .036 \\
\hline Left-right Discrimination & .171 & 1 & 1.049 & 17 & .163 & .691 \\
\hline Name Writing & 5.333 & 1 & .745 & 17 & 7.158 & .016 \\
\hline Phonemes Discrimination & 6.193 & 1 & .695 & 17 & 8.917 & .008 \\
\hline Verbal Correspondences & 1.985 & 1 & .942 & 17 & 2.107 & .165 \\
\hline Vocabulary & .585 & 1 & 1.024 & 17 & .571 & .460 \\
\hline Pictures Memory & 2.489 & 1 & .912 & 17 & 2.728 & .117 \\
\hline Shapes Memory & 7.162 & 1 & .638 & 17 & 11.233 & .004 \\
\hline Sentences Completion & 1.486 & 1 & .971 & 17 & 1.530 & .233 \\
\hline Graphemes Discrimination & 2.741 & 1 & .898 & 17 & 3.054 & .099 \\
\hline Emotionally Reactive & 1.751 & 1 & .956 & 17 & 1.832 & .194 \\
\hline Anxious, Depressed & .197 & 1 & 1.047 & 17 & .188 & .670 \\
\hline Somatic Complaints & .236 & 1 & 1.045 & 17 & .226 & .641 \\
\hline Withdrawn & .091 & 1 & 1.053 & 17 & .086 & .773 \\
\hline Attention Problems & .590 & 1 & 1.024 & 17 & .576 & .458 \\
\hline Aggressive Behavior & 5.028 & 1 & .763 & 17 & 6.589 & .020 \\
\hline Internalizing Problems & .111 & 1 & 1.052 & 17 & .105 & .750 \\
\hline Externalizing Problems & 4.079 & 1 & .819 & 17 & 4.981 & .039 \\
\hline Total Problems & .963 & 1 & 1.002 & 17 & .961 & .341 \\
\hline VIQ & .596 & 1 & 1.024 & 17 & .582 & .456 \\
\hline PIQ & 2.284 & 1 & .924 & 17 & 2.470 & .134 \\
\hline PSQ & 3.825 & 1 & .834 & 17 & 4.588 & .047 \\
\hline FSIQ & 1.867 & 1 & .949 & 17 & 1.967 & .179 \\
\hline
\end{tabular}

Table 2: ANOVA K-means clustering algorithm analysis.

Notes. VIQ=Verbal Intelligence Quotient; PIQ= Performance Intelligence Quotient; PSQ= Processing Speed Quotient; FSIQ= Full Scale IQ.

shown to be statistically significant at all steps to which measurements were added.

As it is shown in table 2, three EDIT-testing variables such as 'Sketching' ( $p=.011)$, 'Name Writing' ( $p=.001)$, and 'Phonemes Discrimination' ( $\mathrm{p}=.015$ ) were statistically significant different between the participants of the two clusters. Adding the ATHINAtesting variables to those of EDIT, five new variables were recorded with statistical significance, such as 'Laterality' ( $p=.035)$, 'Verbal Correspondences' ( $\mathrm{p}=.001)$, 'Shapes Memory' ( $\mathrm{p}=.000)$, and 'Sentences' Completion' ( $\mathrm{p}=.011$ ). The addition of CBCL1 1 $1 / 2-5$-testing variables revealed three new statistically significant variables, like 'Emotionally Reactive' ( $\mathrm{p}=.000)$, 'Aggressive Behavior' ( $\mathrm{p}=.008$ ), and 'Externalizing Problems' ( $\mathrm{p}=.014)$. Finally, adding the WPPSIIII testing variables, one new statistically significant variable, was revealed, 'Processing Speed Quotient' ( $\mathrm{p}=.047)$. 


\section{Discussion and Conclusion}

In this pilot study, we investigated the possibility of identifying specific domains and their complex interactions in the structure of SLD, as early as possible. The results derived from the applied analysis' methods constitute a strong entity of SLD, the components of which were highlighted with stability, throughout the analysis. Through a data analysis schema, a number of variables were recorded as significant, clustering the participants according to their strengths and weaknesses. The obtained results answer the research questions posed to be investigated in this pilot study and enhance the argument that an "ever-expanding model" should be considered as the most reliable source for a comprehensive early diagnosis of SLD, as follows.

The first question to be answered was whether specific dysfunctions in one or more cognitive, language, and socio-psycho-emotional domains indicate the early onset of SLD.

Our study collected the findings of several cognitive studies that have focused on reading and language performance, with attention on the early occurrence of learning difficulties [17]. Our results, in consistence with literature findings, stress the early correlation between the reading mechanism and various attentional deficits $[29,45]$ difficulties in decoding and understanding words due to phonological disorders, as well as deficits both on phonological and visual-spatial memory [46].

As it is generally discussed $[47,48]$, the early identification of children at family risk of SLD is of great importance in defining the precursors of SLD and translating their early complex interactions with effectiveness in later processes of reading and writing. In line with the above, several studies have reported that during the preschool years, SLD is characterised by slow progress and/or problems in:

- Development and production of phonological codes and the processing of linguistic stimuli, phoneme-grapheme correspondences, and semantic appreciation of words [8,15].

- Psychomotor development, including cross-dominance, laterality, left-right discrimination, grapho-motor ability, body shape, spatio-temporal orientation and visual-motor processing [50].
- Development of neural and cognitive mechanisms, such as memory (short-term memory and working memory), visual and auditory perception, speech perception and selective attention $[16,17]$.

- $\quad$ Socio-psycho-emotional development characterized by internalizing and externalizing problems, emotionally reactive, anxious, aggressive behavior, attention problems [51].

Through the second question, we investigated whether an early multi-collector diagnostic model could be used to formulate accurate and comprehensive diagnostic profiles. The data processing scenario, in which multidivergent data fusion were addressed in, was based on the following theoretical aspect: specific early phenotypic clinical characteristics reflecting endophenotypic dysfunctions potentially lead to early diagnostic profiles of children 'at risk' for SLD [6].

Through the applied data clustering techniques an assembly of data were identified and then classified in clusters, each of them was appointed to a SLD or normal case. As it is shown, the dendrogram and the hierarchical cluster analysis illustrated several clusters representing each time the group of children who fulfilled the criterion of SLD (cluster 1) and non- SLD (cluster 2). As it becomes obvious, the data clustering classification represent multiple diagnostic profiles of SLD, commonly characterized by significant difficulties in domains-as it is also well documented in the literature, such as: (a) visual-spatial abilities, (b) cognitive mechanisms, (c) language acquisition, (d) phonological awareness, (e) sociopsycho-emotional development, and (f) intellectual abilities and functioning. However, aiming not only to estimate the prevalence of these domains but mostly to understand their interactions with specific skills, we tested the strength of specific skills and their connectivity with those domains.

Finally, answering the third question, we tested whether all possible underlined endo-phenotypic alterations of the defined domains could lead to difficulties in specific skills and constitute a clinical profile of SLD. As it is shown, a number of variables were kept consistently significant in all created clusters, as follows: 'Sketching' ( $p=.011)$, 'Name Writing' $(p=.001)$, 'Phonemes Dis- 
crimination' ( $\mathrm{p}=.015)$, 'Laterality' ( $\mathrm{p}=.035)$, 'Verbal Correspondences' ( $p=.001)$, 'Shapes Memory' ( $p=.000)$, 'Sentences' Completion' ( $\mathrm{p}=.011$ ), 'Emotionally Reactive' ( $\mathrm{p}=.000$ ), 'Aggressive Behavior' ( $p=.008)$, 'Externalizing Problems' ( $p=.014)$, and 'Processing Speed Quotient' ( $p=.047)$. These findings -being in accordance with the theoretical approaches and the studies presented in the introduction- are consistent with those of others, who consider the role of specific skills of high significance to the acquisition of these domains, the dysfunctions of which are considered suspicious for the early diagnosis of SLD, as follows [53-55]:

- Laterality, sketching, and name writing in the acquisition of visual-spatial abilities,

- Shapes memory and name writing in the acquisition of working memory,

- $\quad$ Name writing, phonemes discrimination, and verbal correspondences in a successful language (phonological awareness, reading, and writing) acquisition,

- $\quad$ Processing speed, and

- Emotionally reactive, externalizing problems, and aggressive behaviour in psycho-emotional development.

The above results seem to consolidate not only the prevalence of the domains that are considered as early precursors of the occurrence of SLD but mainly, the complexity of their interactions, indicating a strong mutual reinforcement between them. Following this assumption, we are possibly able to clarify how specific early phenotypic clinical characteristics reflect endophenotypic dysfunctions and formulate correct clinical profiles with effective interventions, respectively. In this context, the above findings were drawn up in a UML diagram as a potential Use Case scenario (Figure 4), providing some observable and valuable result to clinicians or specialists in SLD, as follows:

- A candidate child is considered as 'at risk' for SLD, whose endophenotypic characteristics include dysfunctions in visualspatial abilities, working memory, language acquisition, and psycho-emotional development

- His/her phenotype presents difficulties in the skills of sketching, writing his/her name, phonemes; discrimination, laterality, verbal correspondences, shapes memory, sentences' completion, processing speed, and presents problems in his/ her socio-psycho-emotional development, as: emotionally reactive, aggressive behaviour, and externalizing problems.
- The therapist uses a variety of measures to distinguish disorders, makes an appropriate differential diagnosis, and categorizes the difficulties according to specific diagnostic criteria that the clinical profile of SLD should fall into.

- All these interactions create a profile managed by the system administrator who analyses the data and provides feedback to the therapist.

The whole process of methods developed for automated early diagnosis of SLD can be generally subdivided into two steps: feature extraction and classification [56]. Through a moving-window analysis, features that characterize the data are extracted. Then, the classification stage is employed with the calculated features to decide whether these diagnostic measures represent SLD manifestations or not.

Based to all the above, it becomes feasible the fruitful construction of an early multifactor model, translational of the complex substrate of SLD that could offer clear diagnosis contributing to early and effective interventions in SLD. Apart from individual fields, the heterogeneity of data involved for SLD diagnosis, increases enormously the complexity, composing a multifactorial problem, with a large variety of data, wherein the significance of each factor is essentially unknown (Institute Eunice Kennedy Shriver National Institute of Child Health and Human Development) (NICHD).

The core argument of this pilot study was that all the different procedures could be merged into an integrated framework of multiple approaches. Adequate methodologies and findings could highlight the strong interactions between specific factors in the background of SLD. However, it should be mentioned that the achievement of this goal used to, and still does demand long and transverse studies as well as clinical work. New aspects of study are constantly emerging, revealing the complexity of the problem and the wide range of its symptoms and extensions. Consequently, to understand all this frame of reference, more and more imperative is becoming the need to decode the role of factors in the causes of the problem. The long-term outcome of breakthroughs and advances in this procedure will meet the current scientific and technological challenge of developing new dimensions in diagnosis as well as interventions in SLD. 


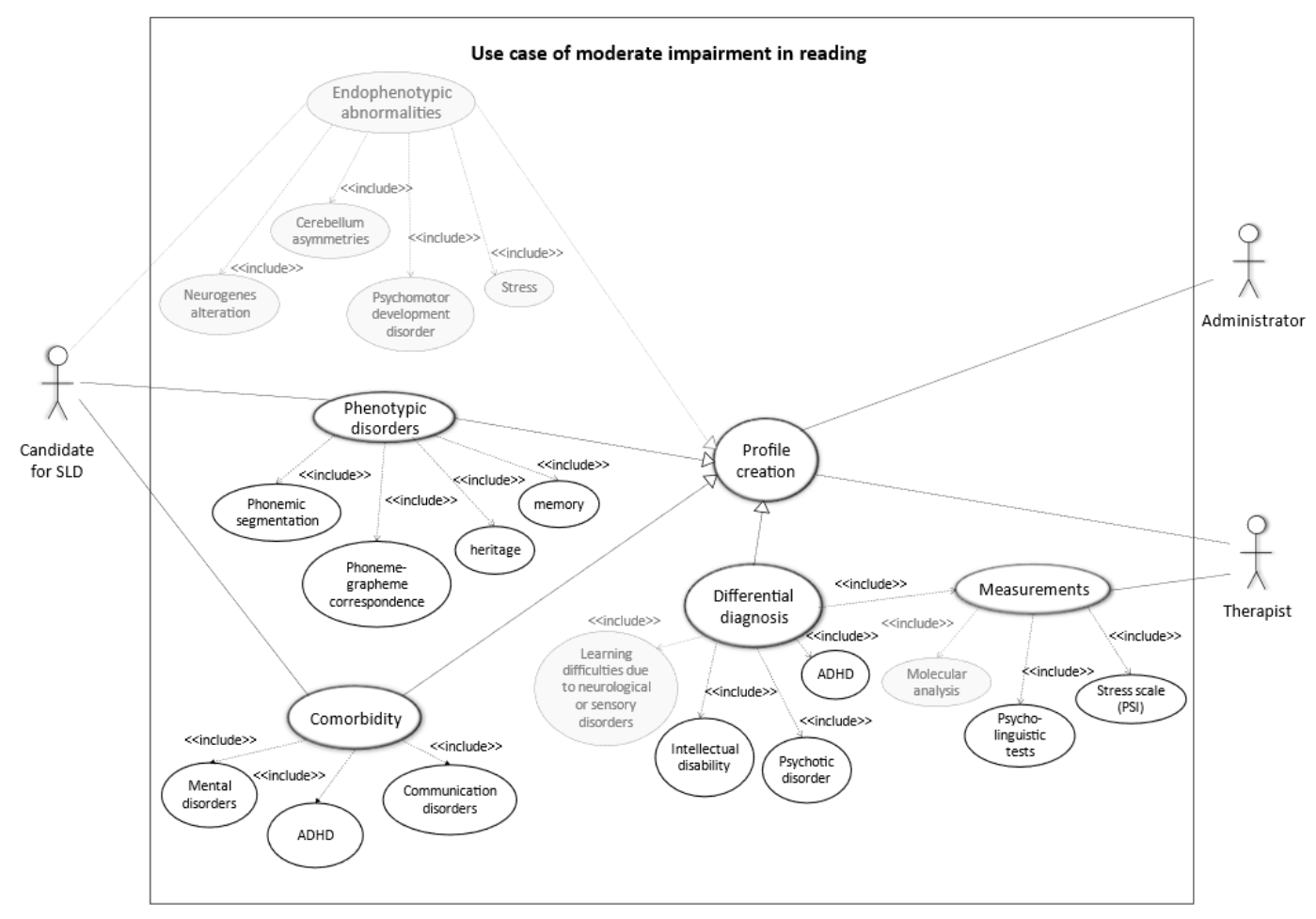

Figure 4: Use case diagram. Fade it out factors are those that have not been examined in the proposed diagnostic procedure.

\section{Limitations}

Limitations of this pilot study need to be noted. First, the inability of including and elaborating data from biological and neurological domains and to be integrated in the analysis model, seems to reduce the strong generalizability of the proposed analysis model. Indisputably, the heterogeneity and complexity of SLD per se, act as a deterrent to the implementation of a universal, unique model of diagnosis. However, in order erroneous or limited evaluations to be prevented, the use of integrated methods (e.g. Big Data) in the diagnostic approach deems to be an entirely requirement. In addition, the restricted sample of kids recruited due to age and general difficulties does not allow us to generalize our results at this point in research. Finally, further research is demanded to integrate as much as possible data (biological and neurophysiological) contributing not only to understand their role in creating safe early diagnostic profiles of SLD, but mostly in creating effective intervention strategies, even at the preschool age.

\section{Conflict of interest}

We have no conflicts of interest to disclose.

\section{Bibliography}

1 Poletti B., et al. "Cognitive-behavioral longitudinal assessment in ALS: the Italian Edinburgh Cognitive and Behavioral ALS screen (ECAS)". Amyotrophic Lateral Sclerosis and Frontotemporal Degeneration 19.5-6 (2018): 387-395.

2 Newbury DF and Monaco AP. "Genetic advances in the study of speech and language disorders". Neuron 68.2 (2010): 309320.

3 Diagnostic and Statistical Manual of Mental Disorders: DSM-5. Arlington, VA: American Psychiatric Association (2013). 
4 Plenty S., et al. "Applying an ESSENCE Framework to Understanding Adult Autism Spectrum Disorder and ADHD: Retrospective Parent Reports of Childhood Problems". The Scientific World Journal (2013): 1-8.

5 Gillberg C. "The ESSENCE in child psychiatry: Early Symptomatic Syndromes Eliciting Neurodevelopmental Clinical Examinations". Research in Developmental Disabilities 31 (2010): $1543-1551$.

6 Fletcher J., et al. "Learning Disabilities: From Identification to Intervention". New York: Guildford Press (2007).

7 Andrande OVCA., et al. "Collective screening tools for early identification of dyslexia". Frontiers in Psychology 5 (2015): $1-13$.

8 Clark KA., et al. "Neuroanatomical precursors of dyslexia identified from pre-reading through to age 11". Brain 137.12 (2014): 3136-3141.

9 Elliott JG and Grigorenko EL. "The Dyslexia Debate". Cambridge: University Press (2014).

10 Mascheretti S., et al. "Neurogenetics of developmental dyslexia: from genes to behavior through brain neuroimaging and cognitive and sensorial mechanisms". Translational Psychiatry 7.1 (2017): e987.

11 Oliver BR and Plomin R. “Twins' Early Development Study (TEDS): A multivariate, longitudinal genetic investigation of language, cognition and behavior problems from childhood through adolescence". Twin Research and Human Genetics 10.1 (2007): 96-1055.

12 Zakopoulou V., et al. "Linking Early Life Hypothalamic-Pituitary-Adrenal Axis Functioning, Brain Asymmetries, and Personality Traits in Dyslexia: An Informative Case Study". Frontiers in Human Neuroscience 13 (2019): 327.

13 Couto JM., et al. "Association of reading disabilities with regions marked by acetylated H3 histones in KIAA0319". American Journal of Medical Genetics. Part B. Neuropsychiatric Genetics 153.2 (2010): 447-462.
14 Tzoufi M., et al. "Early Identification and Intervention in Children with Developmental Dyslexia and Neurological Diseases". Insights on Learning Disabilities 7.1 (2010): 29-47.

15 Norton ES., et al. "Functional neuroanatomical evidence for the double-deficit hypothesis of developmental dyslexia". Neuropsychologia 61 (2014): 235-246.

16 Raschle N., et al. "Pediatric neuroimaging in early childhood and infancy: challenges and practical guidelines". Annals of the New York Academy of Sciences 1252. 1 (2012): 43-50.

17 Xu M., et al. "Atypical lateralization of phonological working memory in developmental dyslexia". Journal of Neurolinguistics 33 (2015): 67-77.

18 Papavasiliou A., et al. "Written language skills in children with benign childhood epilepsy with centrotemporal spikes". Epilepsy and Behavior 6.1 (2005): 50-58.

19 Spironelli C., et al. "Dysfunctional hemispheric asymmetry of theta and beta EEG activity during linguistic tasks in developmental dyslexia". Biological Psychology 77.2 (2008): 123-131.

20 Purcell JJ., et al. "Shared orthographic neuronal representations for spelling and reading". NeuroImage 147 (2017): 554567.

21 Younger JW., et al. "Longitudinal changes in reading network connectivity related to skill improvement". NeuroImage 158 (2017): 90-98.

22 Stein J and Kapoula Z. "Visual Aspects of Dyslexia". Oxford University Press (2012).

23 Ozernov-Palchik $\mathrm{O}$ and Gaab N. "Tackling the Early Identification of Dyslexia with the Help of Neuroimaging". Perspectives on Language and Literacy 42.1 (2016): 11-17.

24 Tammimies K., et al. "Ciliary dyslexia candidate genes DYX1C1 and DCDC2 are regulated by Regulatory Factor X (RFX) transcription factors through X-box promoter motifs". The FASEB Journal 30.10 (2016): 3578-3587. 
25 Papadopoulos TC and Kendeou P. "Investigating the Factor Structure and Measurement invariance of Phonological Abilities in a Sufficiently Transparent Language". Journal of Educational Psychology 104.2 (2012): 321-336.

26 Anthony JL., et al. "Assessment of individual differences in phonological representation". Reading and Writing 23 (2010): 969-994.

27 Verhoeven L., et al. "Cognitive and linguistic factors in reading acquisition". Reading and Writing 24.4 (2011): 387-394.

28 Plakas A., et al. "Impaired non-speech auditory processing at a pre-reading age is a risk-factor for dyslexia but not a predictor: an ERP study". Cortex 49.4 (2013): 1034-1045.

29 Vidyasagar TR and Pammer K. "Dyslexia: a deficit in visuospatial attention, not in phonological processing". Trends in Cognitive Sciences 14.2 (2010): 57-63.

30 Goswami UA. "Temporal sampling framework for developmental dyslexia". Trends in Cognitive Sciences 15.1 (2011): 3-10.

31 Department for Children Schools and Families. Practice Guidance for the Early Years. Foundation Stage. Crown, UK (2008).

32 Dahle AE and Knivsberg AM. "Internalizing, externalizing and attention problems in dyslexia". Scandinavian Journal of Disability Research 16.2 (2014): 179-193.

33 Maughan B and Carroll J. "Literacy and mental disorders". Current Opinion in Psychiatry 19.4 (2006): 350-354.

34 Zoccolotti P., et al. "Editorial: Understanding developmental dyslexia: linking perceptual and cognitive deficits to reading processes". Frontiers in Human Neuroscience 10.140 (2016): 7-9.

35 Zoccolotti P., et al. "Isolating global and specific factors in developmental dyslexia: a study based on the rate and amount model (RAM)". Experimental Brain Research 186.4 (2008): 551-560.
36 van Bergen E., et al. "Dutch children at family risk of dyslexia: Precursors, reading development, and parental effects". Dyslexia 17.1 (2011): 2-18.

37 Franceschini S., et al. "Action video games make dyslexic children read better". Current Biology 23.6 (2013): 462-466.

38 Richards TL and Berninger VW. "Abnormal fMRI connectivity in children with dyslexia during a phoneme task: Before but not after treatment". Journal of Neurolinguistics 21.4 (2008): 294-304.

39 World Medical Association Declaration of Helsinki. "Ethical Principles for Medical Research Involving Human Subjects". JAMA 310.20 (2013): 2191-4.

40 Zakopoulou V. "Early Dyslexia Identification Test (EDIT)". PsycTESTS (2003).

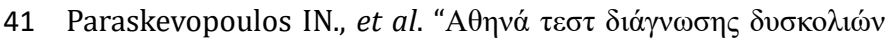
$\mu \alpha ́ \theta \eta \sigma \eta \varsigma$. [Athena Test: Diagnosis of Learning Difficulties]". Ellinika Grammata (1999).

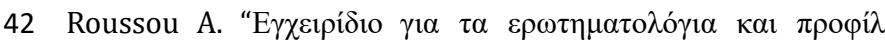

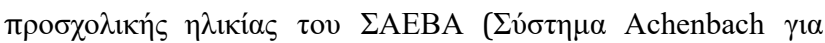

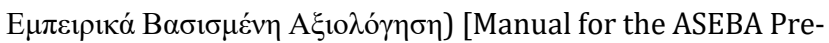
school Forms and Profiles]". Ellinika Grammata (2009).

43 Sideridis G and Antoniou F. "Wechsler preschool and primary scale of intelligence-third edition (WPPSI-III GR)-Standardization in Greek". Topos (2015).

44 Mahalanobis PC. 'On tests and measures of groups' divergence". Journal of the Asiatic Society of Bengal 26.4 (1930): 541-588.

45 Friedmann N., et al. "Developmental attentional dyslexia". Cortex 46.10 (2010): 1216-1237.

46 Alloway TP., et al. "The cognitive and behavioral characteristics of children with low working memory". Child Development 80.2 (2009): 606-621. 
47 Lyytinen H., et al. "Early development of children at familial risk for dyslexia-follow-up from birth to school age". Dyslexia 10.3 (2004): 146-178.

48 Zakopoulou V., et al. "An interpretative model of early indicators of Specific Developmental Dyslexia in preschool age. A comparative presentation of three studies in Greece". Research in Developmental Disabilities 30 (2011): 3003-3016.

49 Wolf U and Gustafsson J. "Structure of phonological ability at age four". Intelligence 53 (2015): 108-117.

50 Zakopoulou, V., et al. "Children 'at Risk' of Specific Learning Disorder: Individualized Diagnostic Profiles and Interventions". Acta Scientific Neurology 4.2 (2021): 25-41.

51 Sorrenti L., et al. "The predicting role of school Learned helplessness in internalizing and externalizing problems. An exploratory study in students with Specific Learning Disorder". Mediterranean Journal of Clinical Psychology 7.2 (2019): 1-14.

52 Mazzocco MM., et al. In Alloway TP. Working Memory and Clinical Developmental Disorders. Routledge. "Working Memory and Specific Learning Disability: Math”. (2018): 106-130.

53 Diamond KE and Baroody AE. "Associations among name writing and alphabetic skills in prekindergarten and kindergarten children at risk of school failure". Journal of Early Intervention 35 (2013): 20-39.

54 Francis DA., et al. "The association between poor reading and internalising problems: a systematic review and meta-analysis". Clinical Psychology Review 67 (2018): 45-60.

55 Horbach J., et al. "Development of behavior problems in children with and without specific learning disorders in reading and spelling from kindergarten to fifth grade". Scientific Studies of Reading 24.1 (2020): 57-71.

56 Tzallas AT., et al. "Epileptic Seizure Detection in Electroencephalograms using Time-Frequency Analysis". IEEE Transactions on Information Technology in Biomedicine 13.5 (2009): 703-710.
Volume 4 Issue 6 June 2021

(C) All rights are reserved by Victoria Zakopoulou., et al. 